\title{
ON THE SHAPES AND SIZES OF CIRRUS PARTICLES CAUSING SPECULAR REFLECTIONS IN LIDAR SIGNALS
}

\author{
Jens Reichardt ${ }^{1, *}$ \\ ${ }^{1}$ Richard-Aßmann-Observatorium, Deutscher Wetterdienst, Am Observatorium 12, 15848 Lindenberg, \\ Germany \\ *Email: jens.reichardt@dwd.de
}

\begin{abstract}
A new method to determine the projection-areaequivalent radius of cirrus particles is presented. Together with direct lidar measurements of ice water content and a simple particle model, the technique facilitates the retrieval of ice particle shape and size. As a first application, cirrus clouds are studied that feature layers with lidar and depolarization ratios highly depressed by mirror reflections from horizontally aligned particles. It is found that in most cases not thin hexagonal plates dominate the ensemble of scatterers but rather compact particles.
\end{abstract}

\section{INTRODUCTION}

Specular reflections of horizontally aligned (2D) cirrus particles cause a decrease in the measured depolarization and lidar ratios. It is widely accepted that these particles are of thin planar shape. However, the measurements with the RAMSES lidar [1] at the Lindenberg Meteorological Observatory in northern Germany, which is equipped with a water spectrometer [2] that facilitates direct measurements of ice water content (IWC), suggest otherwise, because IWC values measured in $2 \mathrm{D}$ cirrus layers are in most cases too high to be explained by the ice volume of large, thin hexagonal plates.

To study this issue further, a new technique was developed to derive the projection-area-equivalent radii in cirrus clouds from the extinction measurements with RAMSES. This information is used as input for a particle model to assess the plausible ranges of particle shape and size of the ice clouds, and thus IWC. By comparing the modeled IWC to the direct RAMSES IWC measurements, one can draw conclusions on the particles that most likely populated the 2D layers. This conference contribution presents first results of the analysis.

\section{METHODOLOGY}

\subsection{Determination of cirrus-particle projection- area-equivalent radius}

RAMSES is equipped with two receivers of different field-of-view (FOV) (0.33 mrad vs. 3.23 mrad) [1] which makes the cirrus extinction measurements with these receivers susceptible to multiple scattering (MS) at varying degrees. Once properly corrected for MS, the obtained singlescattering extinction profile should be the same for both. This identity opens up an elegant method to estimate the projection-area-equivalent radius $\left(r_{\text {pae }}\right)$ of cirrus clouds: For a prescribed cirrus profile of $r_{\text {pae }}$ the MS contributions to the $\mathrm{N}_{2}$ vibrational-rotational Raman signal are calculated with a fast MS code [3] for both FOVs, and the MS-corrected extinction profiles are calculated and compared. The $r_{\text {pae }}$ profile is adjusted and the procedure repeated until satisfying agreement is reached.

As a side note, a similar 2-FOV Raman lidar technique was applied previously to estimate the effective droplet radius of warm clouds [4]. However, the experimental setup differed from RAMSES', in that only a single telescope was used. By drilling a hole in one of the receiver's deflecting mirrors, a narrow-FOV measurement could be obtained in transmission, and a wideFOV measurement in reflection. This approach would probably not be applicable to ice clouds, because the scattering phase function of the comparatively large ice particles is narrowly peaked in the forward direction leaving insufficient signal for the wide-FOV observation.

\subsection{Measurement of ice water content}

Cloud-water Raman backscattering coefficients are measured with the RAMSES water spectrometer [2]. Spectroscopic data [5, 6] are used to convert these coefficients into IWC. Differences in Raman efficiency between cloud 
droplets and ice particles are accounted for. However, IWC measurements are only possible at night, under favorable atmospheric conditions and often only in the lower cirrus ranges because of the extremely weak inelastic return signals from clouds.

\subsection{Retrieval of particle shape and size}

Modeled IWC profile depends on $r_{\text {pae }}$, the particle number concentration, and the assumed shape, orientation and length-to-width (aspect) ratio of the particles. Overfitting is avoided by assuming that all ice particles are identical. The number concentration is obtained directly from the singlescattering extinction-coefficient profile and the particles' geometrical cross section $G=\pi r_{\text {pae }}^{2}$. Random (3D) orientation can be expected when cirrus depolarization and lidar ratios are high ( $>$ $\sim 0.2$ and $>\sim 10 \mathrm{sr}$, respectively), at least partial $2 \mathrm{D}$ orientation otherwise. The basic particle shape is assumed to be hexagonal. Selection of aspect ratios follows loosely Ref. [7]. The set of model parameters is varied until reasonable agreement with the measured IWC profile is reached.

\section{RESULTS}

\subsection{Cirrus with randomly oriented particles}

First, examples of 3D cirrus are discussed. Figure 1 presents two measurement cases on 05 Dec 2015 where the particle optical properties clearly indicate the lack of 2D effects. At 19:00 UTC, MS-corrected extinction profiles show very good agreement for $r_{\text {pae }}=50 \mu \mathrm{m}$, and measured and modeled IWC are alike when 3D columns with an aspect ratio of 2.5 are assumed. Half an hour later, at 19:30 UTC, the cirrus cloud has changed. The center of the cloud is lower, retrieved $r_{\text {pae }}$ is larger $\left(r_{\text {pae }}=80 \mu \mathrm{m}\right)$, and the particle ensemble is probably a mixture of $3 \mathrm{D}$ columns and plates.

\subsection{Cirrus with layers containing horizontally oriented particles}

Next, two cases are examined where the 2D cirrus layers consisted predominantly of thin planar particles. As a matter of fact, it was difficult to find measurements that would be suited as convincing examples. The best candidates are presented in Fig. 2. Lidar and depolarization ratios are decisively smaller than in the flanking 3D

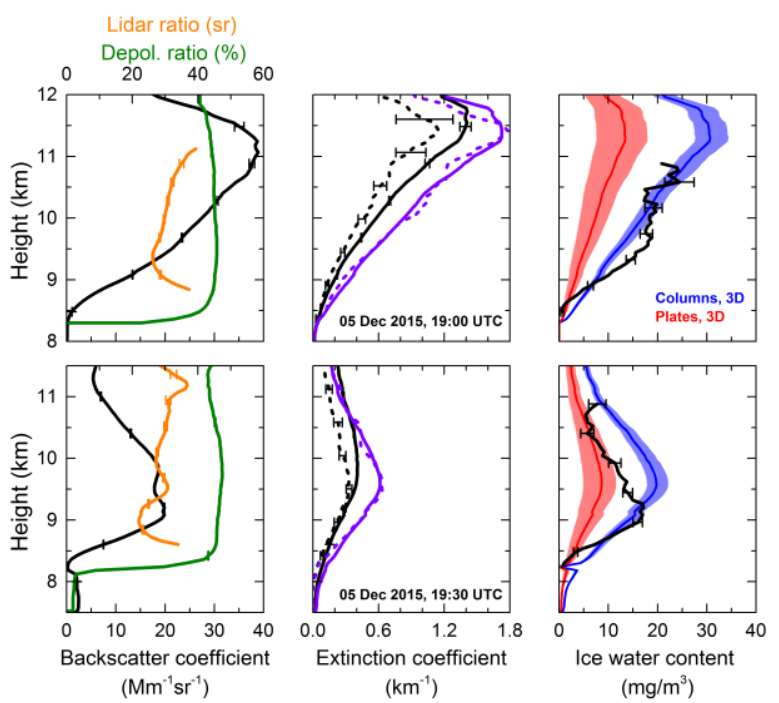

Figure 1. Retrieval results for two observations of 3D cirrus. (left) Particle backscatter coefficient, lidar ratio and particle depolarization ratio. (middle) Particle extinction coefficient as measured with the wide-FOV receiver (black dashed curve) and with the narrow-FOV receiver (black solid curve), and after the correction for multiple scattering (colored curves). (right) Measured (black curve) and retrieved ice water content (colored curves). Either 3D plates or columns are assumed, aspect ratios are 0.08 and 2.5 , respectively. Shaded areas are supposed to illustrate natural variability. Lidar data integration time is 20 min, bars indicate statistical errors. The wide-FOV receiver has a telescope diameter four times smaller than the narrow-FOV receiver, which explains the different noise levels of the extinction profiles.

layers, albeit not extremely low (cf. Fig. 3). Therefore, the effect of mirror reflections is not dominant - perhaps because the fraction of $2 \mathrm{D}$ particles was small, or the oriented particles were poor reflectors (rough surfaces).

In the case of the measurement on 9 Nov 2015, good agreement in the single-scattering extinction profiles could only be achieved below $5.1 \mathrm{~km}\left(r_{\text {pae }}\right.$ $=80 \mu \mathrm{m})$. Still, above $4.5 \mathrm{~km}$ it seems justified to conclude that only a mix of thin $2 \mathrm{D}$ and $3 \mathrm{D}$ planar crystals can explain the measured IWC values. At lower altitudes, however, one has to assume rather compact columns instead.

On 5 Dec 2015, both MS-corrected extinction profiles fit together at all altitudes $\left(r_{\mathrm{pae}}=130 \mu \mathrm{m}\right)$. Again, in the upper part of the 2D layer only thin 


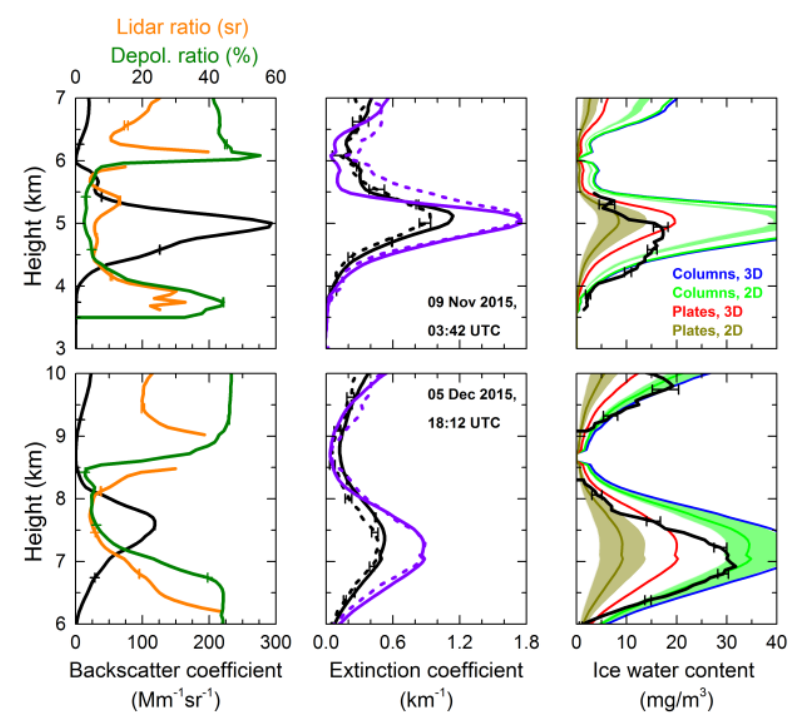

Figure 2. Same as in Fig. 1, but for two observations of 3D cirrus with embedded 2D layer of low ice water content. Either 3D, or 2D, plates or columns are assumed, aspect ratios are 0.06 and 1.5 (top), and 0.08 and 3.0 (bottom), respectively.

plates reproduce the measured IWC values well while elongated columnar particles are required below. Note that the latter are a good particle model for the 3D cirrus above $9 \mathrm{~km}$ as well.

Finally, measurement examples are presented that can be considered characteristic of most 2D cirrus clouds observed with RAMSES (Fig. 3). In all cases, single-scattering extinction profiles agree quite well, derived $r_{\text {pae }}$ values are about 90, 120, and $90 \mu \mathrm{m}$ (Fig. 3, top to bottom). Note that on 21 Dec 2015 the particle backscatter coefficient had rather extreme peak values of more than $1 \mathrm{~km}^{-1} \mathrm{sr}^{-1}$ which, together with miniscule particle depolarization ratios $(\sim 0.02)$ and lidar ratios $(\sim 2$ sr), demonstrate an overwhelming scattering contribution of 2D particles. Only thick plates or compact hexagons are voluminous enough to explain the significant IWC of the layer. Incidentally, for hexagonal particles with aspect ratios around one the geometrical cross section does not depend strongly on orientation which is why $2 \mathrm{D}$ and $3 \mathrm{D}$ IWC profiles are close to each other.

The same conclusion can be drawn from the other two examples depicted in Fig. 3: Thick planar or compact columnar particles are required to reproduce the IWC profiles directly measured

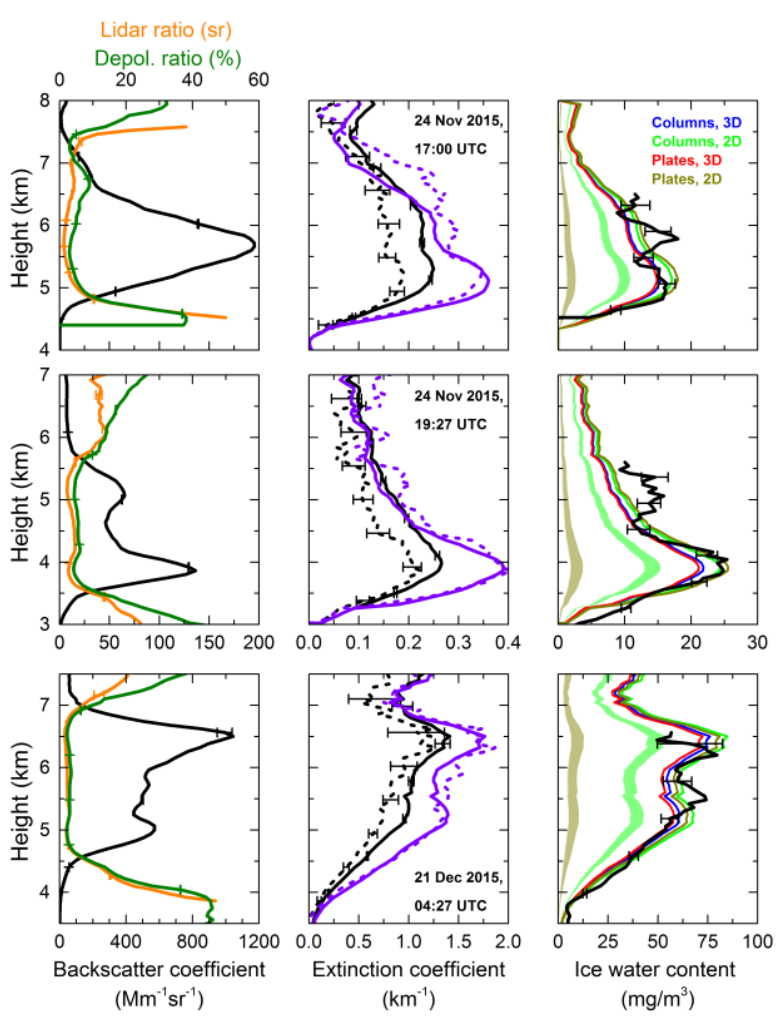

Figure 3. Same as in Fig. 1, but for three observations of 3D cirrus with embedded 2D layer of substantial ice water content. Either 3D, or 2D, plates or columns are assumed, aspect ratios are 0.55 and 1.0 (top, center), and 0.50 and 1.0 (bottom), respectively. Shaded areas illustrate results obtained for thin 2D plates (aspect-ratio range of 0.04-0.08) and elongated 2D columns (2.5-3.0).

with RAMSES while thin plates or elongated columns cannot.

\section{CONCLUSIONS}

The new 2-FOV Raman lidar technique offers a straightforward way to estimate the effective projection-area-equivalent radii of cirrus cloud particles. The method yields robust and reasonable results, for instance retrieved $r_{\text {pae }}$ tends to decrease with height as one would expect. However, because the MS code used employs the small-scattering-angle approximation it is limited to ice clouds with extinction coefficients smaller than $\sim 2 \mathrm{~km}^{-1}$ so that light scattering does not become too diffusive.

From the retrieved values of $r_{\text {pae }}$ and aspect ratio one can calculate the maximum dimension of the 
ice hexagons. For the 2D cirrus layers shown in Fig. 3, maximum dimensions of thick plates range from 200 to $260 \mu \mathrm{m}$, while those of compact columns are somewhat smaller $(165-210 \mu \mathrm{m})$. Because of the poor agreement between retrieved and measured IWC, the results for the probably thin 2D plates of Fig. 2 should be considered inconclusive, but still seem to indicate plate diameters slightly larger than those of the thick plates (Fig. 3). Finally, the elongated 3D columns of the cold cirrus cloud in Fig. 1a have lengths of about $150 \mu \mathrm{m}$, the particle maximum dimension retrieved for the ice cloud of Fig. $1 \mathrm{~b}$ is around 235 $\mu \mathrm{m}$, irrespectively of basic shape.

The main result of this study is that in cirrus layers with the optical characteristics of 2D particles IWC is often considerable, which is inconsistent with the notion that thin planar particles dominate the particle ensemble here. Instead, compact hexagons with aspect ratios around one explain the observations best. This outcome suggests that $2 \mathrm{D}$ cirrus clouds may be better described as being composed of ensembles of compact scatterers that are horizontally aligned, a fraction of which such that they function as mirrors.

Depending on the perfectness of the particle surfaces (and interior), varying fractions may be required to noticeably affect the optical properties. The relation between the flawlessness of the reflecting particle, fraction of 2D particles, and lidar and depolarization ratio is illustrated in Fig. 4 , for the derivation of the underlying equations see Ref. [8]. Cloud optical properties always reach their minima for a $2 \mathrm{D}$ fraction of $100 \%$, and the minimum values themselves can be assumed to be connected with the irregularity of the particles [8]. So the better the optical quality of ice particles the smaller the fraction of $2 \mathrm{D}$ particles acting like mirrors needs to be to obtain a drop to a certain lidar ratio or depolarization ratio. In summary, the encouraging results presented here warrant future studies of 2D cirrus layers. In particular, the quest for convincing measurement examples of ice clouds with horizontally aligned thin planar particles will continue.

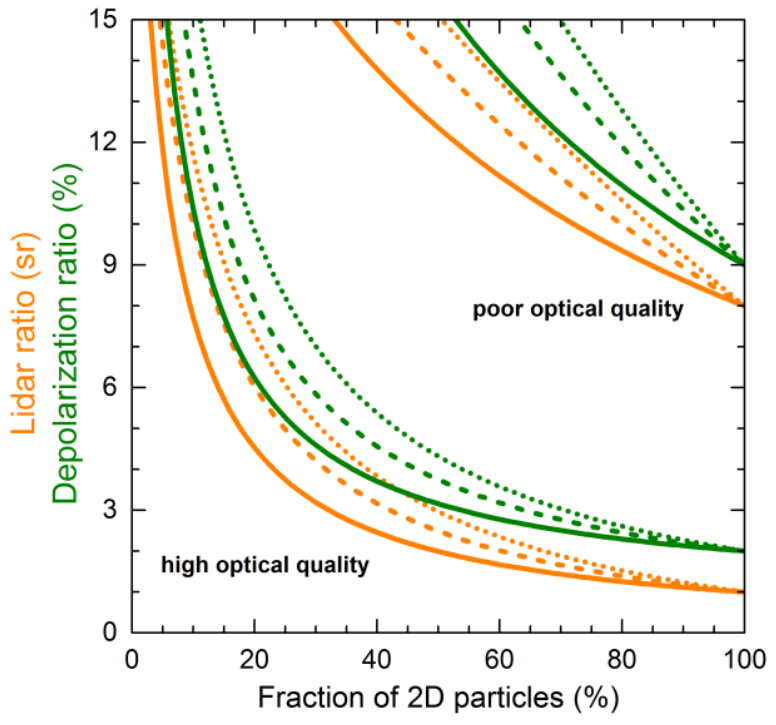

Figure 4. Cirrus lidar ratio and depolarization ratio as functions of the fraction of horizontally aligned ice particles. For the calculations, common 3D values of $25 \mathrm{sr}$ and $40 \%$, respectively, are selected. Assumed 2D values fall in the ranges of the lowest 2D lidar and depolarization ratios ever measured with RAMSES (high optical quality, 1 - 2 sr, 1\% $2 \%)$ or are chosen to illustrate the effect of poor particle optical quality $(8 \mathrm{sr}, 9 \%)$. Results are presented for aspect ratios of 0.5 (solid curves), 1.0 (dashed curves), and 1.5 (dotted curves).

\section{REFERENCES}

[1] J. Reichardt, et al. Appl. Opt. 51, 8111-8131 (2012) [2] J. Reichardt, J. Atmos. Oceanic Technol. 31, 19461963 (2014)

[3] R. Hogan, J. Atmos. Sci. 65, 3621-3635 (2008)

[4] J. Schmidt, et al. Appl. Opt. 52, 2235-2247 (2013)

[5] T. Plakhotnik, et al. J. Quant. Spectrosc. Radiat. Transf. 194, 58-64 (2017)

[6] T. Plakhotnik, et al. J. Quant. Spectrosc. Radiat. Transf. 208, 172-178 (2018)

[7] A. H. Auer, Jr., et al. J. Atmos. Sci. 27, 919-926 (1970)

[8] J. Reichardt, et al. J. Geophys. Res. 107, doi: 10. 1029/2002JD002589 (2002) 